\title{
Über „,Digital Natives“, die selbst aktiv werden und Lehrpersonen, die keine Angst vor Tablets haben. Eine Diskursanalyse zum Schreiben über das Lehren und Lernen mit digitalen Medien in praxisinstruktiven Zeitschriften für die Grundschule
}

\author{
Anna Moldenhauer (D) - Marc Fabian Buck (D) - Thomas Koinzer (D)
}

Online publiziert: 14. Januar 2020

(C) Der/die Autor(en) 2020

Zusammenfassung In diesem Artikel werden die Ergebnisse einer praxeologischen Diskursanalyse zum Schreiben von Grundschullehrer*innen über das Lehren und Lernen mit digitalen Medien in den Fokus gerückt. Auf Basis einer Analyse von Beiträgen aus praxisinstruktiven Zeitschriften für die Grundschule wird in den Blick genommen, welche Begründungsfiguren sowie Subjektpositionen konstituiert werden. Es wird analysiert, wie sich die schreibenden Grundschullehrer*innen als Orientierung vermittelnde Subjekte und als Mitgestalter*innen einer technologisierten und in Teilen gamifizierten Unterrichtspraxis positionieren, in der die generalisierten Schüler*innensubjekte motiviert und selbsttätig lernen.

Schlüsselwörter Digitale Medien · Praxeologische Diskursanalyse · Positionierungen von Lehrer*innen · Lehrer*innenzeitschriften

Jun.-Prof. Dr. A. Moldenhauer $(\bowtie)$ · Prof. Dr. T. Koinzer

Institut für Erziehungswissenschaften, Kultur-, Sozial- und Bildungswissenschaftliche Fakultät, Humboldt-Universität zu Berlin, Unter den Linden 6, 10099 Berlin, Deutschland

E-Mail: moldenha@hu-berlin.de

Prof. Dr. T. Koinzer

E-Mail: thomas.koinzer@hu-berlin.de

Dr. M. F. Buck

Allgemeine, Interkulturelle und International Vergleichende Erziehungswissenschaft sowie Pädagogische Psychologie (EW 1), Fakultät für Erziehungswissenschaft, Universität Hamburg, Von-Melle-Park 8, 20146 Hamburg, Deutschland

E-Mail: marc.fabian.buck@uni-hamburg.de 


\title{
About "digital natives" who become active themselves and teachers who are not afraid of tablets. A discourse analysis on writing about teaching and learning with digital media in teacher journals for primary school
}

\begin{abstract}
This article discusses the results of a praxeological discourse analysis of primary school teachers' writing about teaching and learning with digital media. On the basis of an analysis of contributions from teacher journals for primary school, the focus will be on which legitimations and subject positions are constituted. It will be analysed how the writing primary school teachers position themselves as subjects who are orientating pupils who are co-creators of a technologised and in part gamified teaching practice in which pupils enjoy learning and become active themselves.
\end{abstract}

Keywords Digital media Praxeological discourse analysis $\cdot$ Positioning of teachers $\cdot$ Teacher journals

\section{Problemaufriss}

Die Entwicklung und Förderung digitaler Kompetenzen erfährt bildungspolitisch und medial eine hohe Aufmerksamkeit. Sie wird geradezu zu einer unhintergehbaren Zukunftsherausforderung und Bedingung für gesellschaftliche Teilhabe stilisiert (u.a. KMK 2016). Neben bildungspolitischen Akteur*innen werden nicht zuletzt auch Schulen und Lehrpersonen adressiert (BMBF 2019). Letztere werden aufgefordert, digitale (Bildungs-)Medien vermehrt im Unterricht einzusetzen und digitale Kompetenzen zu vermitteln, die in der KMK-Strategie „Bildung in der digitalen Welt" gar zur vierten elementaren Kulturtechnik neben Lesen, Schreiben und Rechnen erhoben werden (KMK 2016, S. 7 ff.).

Vor dem Hintergrund der hohen Erwartungen an das Lernen und Lehren mit digitalen Medien ist zu fragen, wie Lehrpersonen sich hierzu positionieren bzw. wie sie positioniert werden und wie das Lehren und Lernen mit digitalen Medien im Schreiben entworfen wird. Diese Fragen aufgreifend stellt unser Beitrag die Ergebnisse einer Analyse von Artikeln aus Praxiszeitschriften für Grundschullehrer*innen in den Mittelpunkt der Aufmerksamkeit. Derartige Lehrer*innenzeitschriften stellen ein Forum dar, über welches gesellschaftliche und bildungspolitische Erwartungen in praxisinstruktiver Weise transformiert, verarbeitet und verbreitet werden. Wir gehen der Frage nach, wie Grundschullehrer*innen in Praxiszeitschriften über das Lehren und Lernen mit digitalen Medien schreiben und wie dieses dabei hervorgebracht wird. Dabei interessieren uns sowohl die im Schreiben produzierten Subjektpositionen als auch die Normen und Legitimierungen, welche das Lehren und Lernen mit digitalen Medien - etwa mit interaktiven Whiteboards, Computern oder Tablets -, so wie es in den Zeitschriftenartikeln konstituiert wird, bestimmen.

Zum Aufbau des Artikels: Zunächst resümieren wir den Forschungsstand in Bezug auf Diskursanalysen von Lehrer*innenzeitschriften (2) sowie das Lehren und Lernen mit digitalen Medien (3) und skizzieren das methodische Vorgehen (4). Anschließend stellen wir unsere Ergebnisse dar (5), diskutieren diese unter Rückbezug 
auf den Forschungsstand (6) und benennen Anschlussfragen, die sich aus der vorliegenden Studie ergeben (7).

\section{Diskursanalysen von Lehrer*innenzeitschriften und Ratgebern - die Hervorbringung von Schüler*innen- und Lehrer*innensubjekten in praxisinstruktiven Veröffentlichungen}

In den letzten Jahren sind viele diskursanalytisch ausgerichtete Studien erschienen, welche die Hervorbringung von Wissen und von Subjektivierungsweisen in praxisinstruktiven Zeitschriften und Ratgebern in den Fokus rücken. Gleichwohl sich keine der im Folgenden knapp erwähnten Studien mit dem Lehren und Lernen mit digitalen Medien befasst, sind sie für die vorliegende Analyse insofern interessant, als dass sie die Aufmerksamkeit auf die Produktion von Wissen und von Subjektpositionen in praxisnahen Veröffentlichungen richten. Sie geben damit eine mögliche Kontrastfolie ab, vor deren Hintergrund sich das Schreiben über ein Lehren und Lernen mit digitalen Medien in seiner Spezifik einordnen lässt.

So untersuchen Rabenstein und Reh (2009), wie in einem Ausschnitt aus dem grundschulpädagogischen Diskurs um die Einführung offenen Unterrichts Schüler*innen als selbstständig dargestellt und zugleich Gegenbilder von schwierigen, unaufmerksamen und unselbstständigen Schüler*innen entworfen werden, die eben diesen Erwartungen nicht genügen (Rabenstein und Reh 2009, S. 160). Auch Thon (2014) analysiert Ratgeberliteratur. Statt Formen offenen Unterrichts im Allgemeinen in den Blick zu nehmen, richtet sie den Fokus auf „die Individualisierung von Unterricht". Sie diskutiert Ratgeber als Anrufungsanleitungen - im Sinne Althussers (1977) und Butlers (1997; beide zitiert nach Thon 2014, S. 157) - für Lehrer*innen und arbeitet heraus, wie Lehrer*innen als welche adressiert werden, die ihre Schüler*innen als sowohl different als auch eigenverantwortlich anrufen sollen (Thon 2014, S. 159). Mit der Diskussion um Individualisierung von Unterricht befassen sich auch Klomfaß und Moldenhauer (2018), indem sie Artikel aus der Lehrer*innenzeitschrift PÄDAGOGIK analysieren und der Frage nachgehen, wie schulpädagogisches Wissen um Individualisierung darin konstruiert und kommuniziert wird. Im Ergebnis rekonstruieren sie, wie Individualisierung in diesem Diskursausschnitt als universale Lösungsformel für den „Umgang“ mit Heterogenität diskutiert wird, welche „die Schulpraxis vor handwerkliche Herausforderungen stellt“ (Klomfaß und Moldenhauer 2018, S. 214), die selbst jedoch keiner weiteren Begründung zu bedürfen scheint (Klomfaß und Moldenhauer 2018, S. 226). Während Thon (2014) sowie Klomfaß und Moldenhauer (2018) der Vermittlung eines schulpädagogischen Wissens um Individualisierung nachgehen und Rabenstein und Reh (2009) die Darstellung von Schüler*innen in den Mittelpunkt rücken, analysieren Schmidt und Herfter (2019), wie in Praktiken der Adressierung von Lehrpersonen eine veränderte Aufgabenkultur entworfen und vermittelt wird (ebd.). Schmidt und Herfter (2019) arbeiten vier Figuren heraus, ,in denen je unterschiedlich gefasst wird, was eine (gute) Aufgabe ausmacht und wie über Aufgaben schulische Akteure miteinander ins Verhältnis gesetzt werden" (Schmidt und Herfter 2019, o.S.). Die Figuren lassen sich dahingehend bündeln, dass sie alle eine Norm der Optimierung 
von Unterricht aufrufen. Unterricht bzw. die pädagogische Praxis wird vor diesem Hintergrund als entwicklungsbedürftig ausgewiesen (Schmidt und Herfter 2019).

\section{Lehren und Lernen mit digitalen Medien - zwischen Technologisierung, Critical Digital Citizenship und Gamification}

Unter Berücksichtigung englischsprachiger Veröffentlichungen fällt auf, dass im Zusammenhang mit dem Lehren und Lernen mit digitalen Medien von Selwyn et al. (2016) zwar viele Fragen aufgeworfen werden, die an die hier vorliegenden Forschung anschlussfähig sind ${ }^{1}$, und dass einige Policy-Studien existieren, die auch Positionierungen von Lehrpersonen berücksichtigen (u. a. Philip und Garcia 2013; Pereira und Pereira 2015; Player-Koro et al. 2018), dass bislang jedoch keine Analysen praxisinstruktiver Zeitschriften durchgeführt wurden.

Mit Blick auf wissenschaftliche Studien zum Lehren und Lernen mit digitalen Medien, die im deutschsprachigen Raum veröffentlicht wurden, ist zu konstatieren, dass diese - ähnlich wie es Schmidt und Herfter (2019) für die Diskussion zu unterrichtlichen Aufgaben herausgestellt haben - stark durch die Norm einer Optimierung von Unterricht geprägt sind. Während eine Vielzahl von Publikationen aus der Medien- und der Schulpädagogik sich der Frage widmet, wie Unterricht durch den Einsatz digitaler (Bildungs-)Medien entwickelt und verbessert werden kann (z.B. Matthes et al. 2013; Peschel und Irion 2016; Bastian und Aufenanger 2017; Schaumburg und Prasse 2019), fokussieren vergleichsweise wenige Arbeiten auf grundlegende Transformationen von Unterricht und die mit Schule und Unterricht unter den Bedingungen von Digitalisierung konstituierten Subjektpositionen (Mayrberger 2007; Höhne 2015; Buck 2017; Macgilchrist 2017a, 2017b; aus dem englischsprachigen Raum u. a. Selwyn 2013; Peck et al. 2015; Johnson 2019). Letztere diskutieren Transformationsprozesse des Lehrens und Lernens im Zuge des Einsatzes digitaler Medien unter anderem mit Bezug auf Konzepte von Technologisierung (Höhne 2015), Critical Digital Citizenship (Macgilchrist 2017a) und Gamification (Buck 2017). Sie werden hier kurz umrissen und im Rahmen der Diskussion der nachfolgend dargestellten Rekonstruktionsergebnisse wieder aufgegriffen.

Mit Technologisierung bezeichnet Höhne (2015) unter Rückgriff auf einen mehrdimensionalen Begriff von Technologie, wie ihn auch Foucault sowie Bröckling und Krasmann nutzen (Foucault 1993, S. 23; Bröckling und Krasmann 2010, S. 27 beide zitiert nach Höhne 2015, S. 12), Medientechnologien, Steuerungs- bzw. Kontrolltechnologien und Selbsttechnologien. Er nutzt den Begriff der Technologisierung, um Veränderungen zu fassen, denen Bildungsmedien, wie das Schulbuch unterworfen sind, und hebt hervor, dass Technologisierung (Medien-)Praktiken und Subjektivierungsformen einschließe und Transformationen gesellschaftlicher und pädagogischer Erwartungen an Subjekte evoziere (Höhne 2015, S. 13). Eine Bezugnahme auf Fragen der Technologisierung eröffne eine Vielzahl an Forschungsperspektiven (Höhne 2015, S. 16). So gelte es unter anderem ,die wissensformierende Rolle

\footnotetext{
1 Etwa die Frage nach Transformationen unterrichtlicher und schulischer Praktiken unter den Bedingungen von Digitalisierung.
} 
neuer Medien auf die Veränderung von Schule und Unterricht hin stärker zu berücksichtigen“ (Höhne 2015, S. 16). Eine solche Forschungsperspektive greift auch Macgilchrist (2017a) auf, indem sie die Diskussion um die Strategie der Kultusministerkonferenz „Bildung in der digitalen Welt“ (KMK 2016) in den Blick nimmt und analysiert, welche Subjektfiguren in diesem Kontext entworfen werden. Gemäß Macgilchrist sind es insbesondere drei Figuren, welche die Diskussion dominieren: die Nutzer*innen, die kritisch-reflexiven Subjekte und die Maker $^{2}$ (Macgilchrist 2017a). Drei weitere Figuren - die Expert*innen, die Ecosoph*innen und die Gesellschaftsgestalter*innen ${ }^{3}$ - analysiert sie als marginal und verknüpft sie mit Überlegungen zu einer Critical Digital Citizenship. Die zuletzt genannten Subjektfiguren, dies sei für eine kritische Digital Citizenship entscheidend, seien politische Subjekte (Macgilchrist 2017a, S. 163). Sie ,,reflektieren“ nicht die Gesellschaft, sondern ,beugen' selbstverständliche Erwartungen an die digitale Welt: Diese Figuren ,diffract the rays of technoscience so that we get a more promising interference pattern on the recording films of our lives and bodies“" (Haraway 1997; zitiert nach Macgilchrist 2017a, S. 162). Ähnlich wie Höhne (2015) und Macgilchrist (2017) entwirft auch Buck (2017) eine kritische Perspektive auf bestimmte Facetten einer Transformation von Schule und Unterricht unter Bedingungen von Digitalisierung. Er nutzt dazu das Phänomen der Gamification, mit dem der Versuch beschrieben wird, ,Logik und Elemente des Spiels und ihrer Spielwelten in Praxen zu überführen, die ansonsten andersartigen Prinzipien folgen“ (Buck 2017, S. 270). Buck führt aus, dass Gamification in vielerlei Hinsicht tradierten Ideen von Schule - u. a. als von der Lebenswelt unterschiedener und sie potentiell erweiternder und kontrastierender Ort - entgegenstehe (Buck 2017, S. 271, S. 273).

Die Perspektiven der Technologisierung, der Critical Digital Citizenship wie auch der Gamification sind für die vorliegende Studie von Interesse, da sie in unterschiedlicher Weise mit Digitalisierung verknüpfte Subjektpositionen und Subjektivierungsweisen analysieren und kritisch diskutieren. Sie tun dies vorrangig einerseits mit Blick auf Veränderungen von (Bildungs-)Medien (Höhne 2015) und das Generationenverhältnis (Buck 2017) sowie andererseits auf der Ebene bildungspolitischer Programmatiken und Steuerung (Höhne 2015; Macgilchrist 2017a). Keine der genannten Studien widmet sich explizit den Subjektivierungen und Positionierungen

\footnotetext{
2 Nutzer*innen bedienen vorhandene Technologien, rezipieren Medienangebote und reagieren auf Wandlungsprozesse (Macgilchrist 2017a, S. 148). Kritisch-reflexive Subjekte reflektieren und bewerten die Gefahren und Risiken der Digitalisierung sowie der eigenen Medienanwendung (Macgilchrist 2017a, S. 151); sie tun dies im Sinne einer Medienkritik (Macgilchrist 2017a, S. 152). Die Maker gestalten Inhalte und nutzen digitale Medien für kreative Prozesse (Macgilchrist 2017a, S. 153).

3 Mit den Expert*innen rekurriert Macgilchrist (2017a) auf eine Subjektfigur, die sie in einer Stellungnahme des Deutschen Studentenwerks analysiert hat, in der gefordert wird, Studierende mit Beeinträchtigungen als Expert*innen in eigener Sache einzubeziehen. Als Ecosoph*innen bezeichnet sie ökologisch reflektierte Personen, ,die sich der Komplexität der Mensch-Natur-Beziehungen sowie der eigenen (anthropogenen) Rolle[n] bei der Zerstörung der Umwelt bewusst [sind] und entsprechend handel[n]“ (Macgilchrist 2017a, S. 158). Die Figur der Gesellschaftsgestalter*innen - so Macgilchrist - ähnelt derjenigen der Expert*innen, da hinsichtlich beider Figuren davon ausgegangen wird, dass Schüler*innen kompetent mitgestalten können. Die Mitgestaltung von Welt steht also im Zentrum der Figur der Gesellschaftsgestalter*innen.
} 
von Lehrpersonen, insofern handelt es sich bei der nachfolgend dargestellten Diskursanalyse um eine neue und ergänzende Perspektive.

\section{Methodisches Vorgehen - eine praxeologische Diskursanalyse von Lehrer*innenzeitschriften}

Als Datengrundlage für unsere Diskursanalyse dienen ausgewählte Artikel der Praxiszeitschriften „Grundschule“ sowie „Praxis Grundschule“ seit Beginn des Jahrtausends. Beide Zeitschriften werden seit Jahren breit rezipiert und können als wichtiger Spiegel grundschulischen Sprechens bzw. Schreibens über Unterricht und Schulalltag begriffen werden ${ }^{4}$. Wir beziehen solche Artikel und Themenhefte ein, die von Lehrpersonen für Lehrpersonen geschrieben werden und in denen es um Schule und Unterricht unter den Bedingungen von Digitalisierung geht. Dem Selbstverständnis der Zeitschriften zufolge enthalten die Artikel „Rat auf Augenhöhe“ (Grundschule 2019, o.S.). Sie stellen eine Form der Vermittlung bzw. Kultivierung von Wissen für die Schulpraxis dar (Schmidt und Herfter 2019; Klomfaß und Moldenhauer 2018). Die schreibenden Lehrpersonen legen ihren Kolleg*innen bestimmte Formen des Umgangs mit digitalen Medien nahe und machen diese damit wahrscheinlicher. Am Ende dieses Artikels geben wir eine Übersicht über den von uns analysierten Korpus, der aus insgesamt 15 Artikeln besteht, die seit Beginn des Jahrtausends veröffentlicht wurden. Es handelt sich um sämtliche Artikel, die unter Berücksichtigung der hier dargestellten Kriterien in den beiden genannten Zeitschriften erschienen sind. Nicht einbezogen haben wir solche Artikel, in denen sich Wissenschaftler*innen, Journalist*innen oder Medienberater*innen äußern.

Die ausgewählten Artikel verstehen wir als Fragmente einer diskursiven Praxis, in der das Lehren und Lernen mit digitalen Medien in der Grundschule über diskursive Praktiken auf spezifische Weise konstituiert wird. Wir nehmen an, dass diskursive Praktiken insofern performativen Charakter haben, als dass in jedem Äußerungsakt eine Ordnung postuliert wird, die potenziell (machtvolle) Wirkungen zeitigen kann (Wrana 2015a; Proske und Nissen 2017). Indem fortlaufend Relationierungen vorgenommen werden, wird Sinn konstituiert und werden sowohl Subjektpositionen als auch die Gegenstände der Diskurse - in unserem Fall das Lehren und Lernen mit digitalen Medien - hervorgebracht (Wrana 2015b, S. 125).

Zugleich begreifen wir Sinnkonstitution als sich im Kontext von Kontingenz und Überdetermination vollziehenden Prozess (u. a. Schmidt und Herfter 2019; Wrana 2015a, 2015b). D.h. nicht zuletzt, dass das Schreiben über das Lehren und Lernen mit digitalen Medien auf unterschiedliche Weise möglich ist und verschiedenen Ordnungen unterliegen kann. Bezogen auf die Konstitution von Subjektpositionen

\footnotetext{
4 So erscheint die Praxis Grundschule mit einer Auflage von 12.000 Stück und die Grundschule mit einer Auflage von 5000 Stück (Mediadaten der Zeitschriften - letzter Abruf 03.06.2019). Die Grundschule schreibt von sich: „Während andere pädagogische Fachzeitschriften Inhalte vorrangig aus universitärer Perspektive aufbereiten, geht Grundschule von den konkreten Fragen und Erfahrungen der LehrerInnen aus" (Grundschule Mediadaten 2019, o.S., Hervorhebung im Original). Praxis Grundschule hebt demgegenüber hervor, ,nicht nur eine Fachzeitschrift, sondern ein Medienpaket“ (Praxis Grundschule Mediadaten 2019) zu sein.
} 
resultiert aus dieser Überdetermination, dass erst ,die Vielstimmigkeit des Diskurses [...] den Raum [eröffnet], in dem durch die artikulatorischen Praktiken eine Positionierungsbewegung vollzogen werden kann“" (Wrana 2015b, S. 127). Da jeder Akt der Positionierung „das Moment eines diskursiven Kampfes um die Gültigkeit von Wissensordnungen ist“ (Wrana 2015b, S. 129), rekonstruieren wir, wie Differenzen und Relationen produziert werden und wie das Wissensfeld „Lehren und Lernen mit digitalen Medien in der Grundschule“ in den von uns fokussierten diskursiven Praktiken konstituiert und mit Gültigkeit versehen wird. Diese Perspektive macht den spezifischen Zugang und Erkenntnisgewinn unseres diskursanalytischen Vorgehens aus.

Nach einer ersten Sichtung und groben Sortierung des Korpus, haben wir ausgewählte Aussagen einer Feinanalyse unterzogen. Neben prozeduralen Aspekten haben wir die in diesen Aussagen enthaltenen figurativen und positionalen Aspekte analysiert (Wrana 2015a, S. 121). Die einleitend genannten Fragen nach Subjektpositionen, Normen und Legitimierungen, die im Schreiben konstituiert werden, dienten als Heuristik, um Positionierungen zu fassen (Wrana 2015b, S. 137). Unsere Analyseergebnisse haben wir schließlich mit den anderen im Korpus enthaltenen Texten konfrontiert und verglichen, um „Verdichtungen“ zu identifizieren (vgl. Schmidt und Herfter 2019, o.S.). Die identifizierten Verdichtungen nutzen wir im Folgenden, um unsere Ergebnisse darzustellen.

\section{Zur Rekonstruktion des Schreibens über einen Unterricht mit digitalen Medien}

Wir beginnen die Ergebnisdarstellung mit einem Blick auf die angeführten Begründungsfiguren (a). Anschließend rücken wir die entworfenen Subjektpositionen von Lehrenden und Lernenden in den Mittelpunkt unserer Ausführungen (b) und gehen schließlich auf die Frage ein, inwiefern es im Laufe der von uns betrachteten Zeit zu Verschiebungen gekommen ist (c).

a) Begründungsfiguren

Der Einsatz digitaler Medien im Unterricht wird maßgeblich damit begründet, dass digitale Medien allgegenwärtig seien und einen selbstverständlichen Teil der Lebenswelt oder gar Lebenswirklichkeit von Kindern darstellten (Gach 2018, S. 6; Nuxoll 2018; Thielbeer 2018, S. 20; Stolz 2012, S. 27; Seidel 2011, S. 30; Bongardt 2003, S. 18; Halbach 2003, S. 24). Die schreibenden Grundschullehrer*innen appellieren an Kolleg*innen, diese „Realität“ (Halbach 2003, S. 24) nicht zu verkennen und Fragen der Digitalisierung ,zukunftsweisend“ (Gach 2018, S. 6) zu behandeln. Produziert wird in diesem Zusammenhang die Unterscheidung zwischen einem Unterricht der entweder realitätsfremd und künstlich an Kindern vorbeiarbeitet oder - sofern digitale Medien eingesetzt werden - Identifikations- und Verständigungsgrundlagen bietet. Unterricht gilt dann als gut, wenn in ihm Bezug auf die Lebenswelt der Kinder genommen wird. Oder anders formuliert: Weil digitale Medien Teil der Lebenswelt der Kinder sind, sollten sie auch in der Schule bzw. im Unterricht genutzt werden. 
Eine weitere Variante der Legitimierung wird hervorgebracht, indem frühere Varianten der Nutzung von Medien im Unterricht mit aktuellen Varianten verglichen werden und auf diese Weise eine Differenz zwischen gestern, heute und morgen produziert wird. Digitale Medien werden als Fortschritt gefeiert, den es nicht zu hinterfragen gelte, bei dem es um Fragen der Optimierung von Unterricht durch die „Vorteile“ gehe, welche ,diese neue Technik“ mit sich bringe (Thielbeer 2018, S. 23). So schreibt eine Grundschullehrerin: „Traditionellerweise werden in der Grundschule Plakate zu den Referaten erstellt. [...] Dank der Tablets gehen wir noch einen Schritt weiter“ (Verena ${ }^{5}$ 2018b, S. 46). Andere setzen auf die Hervorhebung eines vereinfachten Einsatzes und die Abgrenzung gegenüber früheren Zwängen: „Lehrkräfte müssen keine Lehrfilme mehr als Filmrollen in große Projektoren einlegen und vorher ausleihen. Stattdessen ruft man in der Schule das Internet auf und sucht auf YouTube den entsprechenden Film“ (Gach 2018, S. 7). Bei Nutzung eines interaktiven Whiteboards ,,muss man sich als Lehrkraft aber nicht über Tastatur und Maus am Computer beugen“" (Seidel 2011, S. 30). In Bezug auf den Einsatz digitaler Medien wird eine Aufbruchstimmung vermittelt und auch damit der Neuigkeitswert unterstrichen: „Es geht los!“ (Verena 2018a/7, S. 46).

Eine dritte Figur, die in unterschiedlichen Formen rekonstruiert werden konnte, über die der Einsatz digitaler Medien legitimiert wird, ist die Annahme, dass digitale Medien neue Formen eigenaktiven, produktiven, selbstverantwortlichen, individualisierten oder gar selbstbestimmten Lernens ermöglichten (Thielbeer 2018, S. 20; Maurer 2016, S. 34; Krause 2007, S. 22; Daugs 2004, S. 6; Bongardt 2003, S. 18; Halbach 2003, S. 24). Internet-Recherchen würden ,in sich die notwendige Individualisierung und Binnendifferenzierung“ bergen, ,da jedes Kind nach seinen bevorzugten Lerneingangskanälen forschen und Texte nach eigener Lesekompetenz auswählen“ könne (Daugs 2004, S. 6). Auffällig, wenngleich wenig überraschend, ist, dass dabei allein Möglichkeiten hervorgehoben und Risiken oder Schwierigkeiten nicht thematisiert werden. Hard- und Software gelten dann als gut, wenn sie intuitiv und selbstständig von den Grundschüler*innen genutzt werden können (Verena 2018b, S. 46; Maurer 2016, S. 34; auch Klöckener 2013).

\section{b) Positionierung der Beteiligten}

Die Positionierung der schreibenden Grundschullehrer*innen selbst kennzeichnet diese als für die Vermittlung von Orientierungswissen unverzichtbar (Bongardt 2003, S. 18; Halbach 2003, S. 24f.; Sawischlewski 2003, S. 58f.; Seidel 2011, S. 31; Gach 2018, S. 7; Nuxoll 2018, S. 38). Nuxoll beispielsweise beschreibt sich und seine Grundschulkolleg*innen als bedeutsam für eine pädagogische Begleitung der Schüler*innen beim Aufwachsen in einer digitalen Welt (Nuxoll 2018, S. 39). Wenngleich die Kinder als solche dargestellt werden, die digitale Medien vielfach ohne Probleme und von Beginn an bedienen könnten (z. B. Verena 2018a, S. 46), wird wiederholt betont, dass es unabdingbar sei, dass Lehrpersonen sie aufklärten (Gach 2018), ihnen einen ,verantwortungsbewussten Einsatz“ nahebrächten (Seidel 2011, S. 31) und ihnen Medienkompetenz vermittelten (Halbach 2003, S. 24; Seidel 2011, S. 31). Es wird damit eine Differenz produziert zwischen der Handhabung

\footnotetext{
${ }^{5}$ Diese Lehrerin schreibt ausschließlich unter ihrem Vornamen.
} 
bzw. Bedienung digitaler Medien auf der einen Seite und einem mündigen, verantwortungsbewussten oder reflektierten Umgang mit denselben und mit den Informationen, die sie verfügbar machen, auf der anderen Seite. Zugleich wird auf Formate wie den Computer- oder Tabletführerschein gesetzt, mithilfe derer die Bedienung der Geräte nicht nur als vermittlungsrelevant, sondern auch als zu zertifizierende gerahmt wird (Sawischlewski 2003; Verena 2018a).

Im Vergleich der Thematisierung verschiedener Medien variieren die Positionierungen sehr weitreichend: So wird in den Ausführungen von Seidel das Bild einer Lehrperson entworfen, die wie ein Animateur vor einem Publikum agiert und - dem interaktiven Whiteboard sei Dank - ,die Aufmerksamkeit der Schüler steuern, Äußerungen anregen und inhaltliche Betonungen im Unterricht setzen“ kann (Seidel 2011, S. 33). Andere setzen darauf, Überzeugungsarbeit zu leisten und positionieren die Kolleg*innen damit als solche, denen nur gut zugeredet werden müsse, damit sie sich trauten, digitale Medien einzusetzen. Sich selbst entwerfen die schreibenden Lehrpersonen als Avantgarde, welche die eigenen - ausschließlich positiven - Erfahrungen mit dem Einsatz digitaler Medien teilten, um Ängste zu nehmen. So titelt die Ausgabe 7/2018 der „Grundschule“ etwa „Keine Angst vor Tablet und Co“ und Nuxoll fordert darin: „Lehrkräfte müssen nicht immer digital unterwegs sein, um die Schüler auf eine digitale Welt vorzubereiten. Wichtig ist, dass sie digitale Bildung als Aufgabe annehmen und angehen“ (Nuxoll 2018, S. 39). Antizipiert wird in diesem Kontext ein verändertes Verhältnis zu Schüler*innen, denen zugeschrieben wird, sich besser mit der neuen Technik auszukennen.

Über die Kinder, Schüler*innen oder „Digital Natives“ (Nuxoll 2018, S. 38) wird in stark generalisierter Form geschrieben. Zwar wird die Metapher der ,Digital Natives“ (ebd.) nur von einer der schreibenden Lehrpersonen explizit verwendet, damit verbundene Figuren von Kindern und Jugendlichen, die digitale Medien alltäglich, selbstverständlich und hinsichtlich der Bedienung kompetent nutzten, werden in den Artikeln jedoch wiederholt in analoger Weise aufgerufen und bearbeitet ${ }^{6}$.

Von den Schüler*innen wird angenommen, dass sie der Einsatz digitaler Medien stets motiviere (u.a. Thielbeer 2018, S. 23; Verena 2018a, S. 46, 2018b, S. 46f.; Seidel 2011, S. 30; Krause 2007, S. 22; Grömping und Schwarze 2003, S. 10f.; Sawischlewski 2003, S. 58). Mit Verweis auf die Freude, die Schüler*innen an der Arbeit mit digitalen Medien hätten, wird der Einsatz derselben legitimiert und eine Norm aufgerufen, der zufolge Lernen freudvoll sein sollte und Lehrpersonen die Schüler*innen bestenfalls motivierten. Verena berichtet: „Die Kinder haben großen Spaß an der Recherche und Präsentation. So kommt es durchaus vor, dass jemand ein freiwilliges Referat über sein Lieblingsthema präsentieren möchte“ (Verena 2018b, S. 47). Und auch Seidel hebt hervor, dass , die Schülerinnen und Schüler [...] motiviert bei der Sache“ (ebd. 2011, S. 33) seien und „Freude an der Arbeit mit der elektronischen Tafel“" hätten (Seidel 2011, S. 30).

Neben der Betonung von Motivation und Freude auf Seiten der Schüler*innen wird denselben zugeschrieben, oftmals nur „diffuses Halbwissen“ (Sawischlewski

\footnotetext{
6 Wir nutzen diese Metapher in der Überschrift des Artikels und im Folgenden daher nicht, um eine*n einzige*n Autor*in zu zitieren, sondern um die Figur darzustellen, mit der sich viele Autor*innen auseinandersetzen. Die Anführungszeichen markieren eine Distanzierung von dieser Figur.
} 
2003, S. 58) zu haben oder den Computer ,nur als Spielzeug “ zu kennen (Stolz 2012, S. 27). Es wird darauf hingewiesen, wie wichtig es sei, den Schüler*innen Orientierungswissen zu vermitteln und sie insbesondere hinsichtlich des Datenschutzes zu sensibilisieren. Auf diese Weise werden tradierte Lehrer*innen-Schüler*innenVerhältnisse nicht infrage gestellt und die Annahme entkräftet, „Digital Natives“ seien Lehrpersonen überlegen.

Eine Infragestellung des Lehrer*innen-Schüler*innen-Verhältnisses geht allerdings von Nuxolls Ausführungen aus. Nuxoll hält fest, ,,dass Schüler das Feedback der Software manchmal eher annehmen als das der Lehrkraft" (Nuxoll 2018, S. 39). Er begründet dies damit, dass das Feedback einer Software objektiv wirke und von den Schüler*innen „emotionslos“ befolgt werde (ebd.). Indem er einen ausdrücklichen Vergleich zwischen dem Zeigen durch Grundschullehrer*innen und dem der Software anstrengt, spitzt er zu, was an anderer Stelle etwa über die Betonung des selbstständigen Arbeitens der Kinder (z. B. Halbach 2003, S. 24f.) und damit verbundenen Verschiebungen und Auslassungen von Lehrtätigkeiten ebenfalls mitschwingt.

Das Lehren mit digitalen Medien rückt in vielen Aussagen in den Hintergrund. Es wird allenfalls als spielerische Quizveranstaltung (Sawischlewski 2003), als Aufbereitung von Tätigkeits- oder Kompetenzbeschreibungen in einer Checkliste (u. a. Verena 2018a) oder als die Blicksteuerung eines Publikums (Seidel 2011) angesprochen. Lernen wird dazu passend mit der Eigenaktivität von Schüler*innen oder Formen des Spiels (Quiz, Detektivarbeit, z.B. Sawischlewski 2003) gleichgesetzt. Teilweise werden selbst Prüfungsaufgaben den Schüler*innen überantwortet und die Einmischung von Seiten der Lehrpersonen aufgrund des zuvor aufgerufenen Lernziels ,selbstständiger Umgang“ als unerwünscht deklariert: „Eine Prüfung der Fähigkeiten durch den Lehrenden ist nicht notwendig. Gerade weil die Kinder den selbstständigen Umgang mit dem Computer lernen sollen, ist es paradox, am Ende der Einheit oder einzelner Stunden eine Demonstration des derzeitigen Lernstandes von ihnen zu verlangen zumal die Ergebnisse der Arbeit bereits einem Partner vorgeführt wurden" (Sawischlewski 2003, S. 59).

c) Verschiebungen im Zeitverlauf

Hinsichtlich der Frage nach Verschiebungen im Zeitverlauf ist zu beachten, dass zwischen 2003 und 2018 grundlegende Veränderungen der technischen Voraussetzungen stattgefunden haben und die digitalen Medien selbst, über welche 2003 geschrieben wurde, möglicherweise nur eingeschränkt mit heutigen Medien vergleichbar sind. Da die vorliegende Analyse nach der Hervorbringung von Normen und Legitimierungen sowie Subjektpositionen im Hinblick auf das Lehren und Lernen mit digitalen Medien fragt und den Fokus folglich nicht auf die digitalen Medien selbst, sondern auf Sinnkonstitutionsprozesse richtet, verzichten wir auf eine medien- und bildungshistorische Kontextualisierung.

Verschiebungen lassen sich in den von uns fokussierten Aussagen insofern beobachten, als dass sowohl Begründungsfiguren als auch Positionierungen in der 
Iteration $^{7}$ Transformationen unterliegen. Diese Verschiebungen fallen allerdings nicht stetig aus, so dass sie sich nicht als Tendenzen beschreiben lassen. Vielmehr zeigt sich eine Pluralität von Figuren der Legitimierung und Positionierung. Zwar lassen sich ähnliche Figuren als Verdichtungen identifizieren, doch kommt es wiederholt auch zu Brüchen und Widersprüchen - etwa, wenn Lehrpersonen einerseits in ihrer Relevanz für die Vermittlung von Orientierung angerufen werden (Bongardt 2003, S. 18; Halbach 2003, S. 24f.; Sawischlewski 2003, S. 58f.; Seidel 2011, S. 31; Gach 2018, S. 7; Nuxoll 2018, S. 38), diese Orientierung jedoch vermittels eigenverantwortlichen Arbeitens oder im Spiel angebahnt werden soll (u. a. Sawischlewski 2003). Verschiebungen entstehen im Schreiben darüber hinaus dadurch, dass zeitliche Differenzen konstituiert werden, die Formen des Fortschrittes nahelegen: alt vs. neu, früher vs. heute usw. (u. a. Gach 2018).

\section{Normative Einsätze eines Schreibens über das Lehren und Lernen mit digitalen Medien in der Diskussion}

Obgleich das produzierte Wissen in vielerlei Hinsicht durchaus als plural beschrieben werden kann, werden die analysierten Verdichtungen im Folgenden bezüglich der zugrundeliegenden normativen Ordnungen zugespitzt und in ihrem Verhältnis zu den zuvor referierten Studien diskutiert. Dabei wird die Komplexität des Geschehens deutlich; das Wissensfeld „Lehren und Lernen mit digitalen Medien“ unterliegt verschiedenen Ordnungen die im Ringen um Gültigkeit unterschiedlich aufgerufen werden und einen Raum für variable Positionierungen eröffnen (Wrana 2015b).

Dass die Entwicklung neuer Medien in vielen Bereichen mit gesellschaftlichen Veränderungen einhergeht und dass daraus auch für die Schule neue Anforderungen resultieren, scheint unstrittig. Auffällig ist allerdings, dass im Schreiben über das Lehren und Lernen mit digitalen Medien ein Bild von Unterricht entworfen wird, dass diesen über Lebensweltnähe und Fortschritt legitimiert. Dabei wird nicht zwischen einem Lernen mit und einem Lernen über Medien differenziert (Bastian 2017). Stattdessen werden Unterscheidungen zwischen einem realitätsfremden, überkommenen und einem durch Unmittelbarkeit gekennzeichneten, zukunftsweisenden Unterricht konstituiert. Die Argumentationen ähneln diesbezüglich - so auch Buck (2017) - denen von Vertreter*innen reformpädagogischer Ansätze.

Auch der Bezug auf Eigenaktivität und Selbstbestimmung legt einen Vergleich zu reformpädagogischen Argumentationen nahe, insofern damit ein verändertes Generationenverhältnis angeregt wird, das die Asymmetrie zwischen Lehrenden und Lernenden verklärt ${ }^{8}$. Zugleich zeigen sich Parallelen zum Schreiben über Unterricht im Modus der Individualisierung (Thon 2014; Klomfaß und Moldenhauer 2018). Den Darstellungen der schreibenden Grundschullehrer*innen zufolge verspricht das Lehren und Lernen mit digitalen Medien eine Lösung für eine Vielzahl unterschiedlicher unterrichtlicher Herausforderungen. Es erscheint geradezu als Technologie

\footnotetext{
7 D.h. in nicht-identischen Wiederholungen von Äußerungsakten, die nicht der Intentionalität von Subjekten unterliegen, sondern der Ordnung einer sozialen Praxis (Wrana 2006, S. 130).

${ }^{8}$ Für weitere Ausführungen zur Reformpädagogik verweisen wir auf Tenorth (2017).
} 
(Höhne 2015), die das viel diskutierte „Technologiedefizit der Erziehung“ (Luhmann und Schorr 1982) überwindbar machen könnte (vgl. auch Philip und Garcia 2013, die diese Erwartung u. a. mit Bezug auf Skinner [ebd. 1961 zitiert nach Philip und Garcia 2013] diskutieren).

In Fortführung einer „Normalisierung der selbstständigen Schülerin“ (Rabenstein und Reh 2009) gelten solche Varianten des Einsatzes digitaler Medien als wünschenswert, die ebenjene Selbstständigkeit und Eigenaktivität zulassen und einfordern und zugleich eine Entlastung der Lehrer*innen von Verantwortung versprechen. Im Sinne der von Höhne (2015) mit Bezug auf Foucault thematisierten Selbsttechnologien, werden Lehrpersonen im analysierten Diskursausschnitt angerufen, keine Angst vor digitalen Medien zu haben und deren Einsatz als (Entwicklungs-)Aufgabe und Notwendigkeit einer Optimierung von Unterricht anzunehmen (Nuxoll 2018; kritisch: Thon 2014; Klomfaß und Moldenhauer; Schmidt und Herfter 2019).

Die Kontrastierung der von Macgilchrist (2017a) rekonstruierten Subjektfiguren mit den im Schreiben von Grundschullehrer*innen konstituierten Positionen verweist auf eine Reihe von Ähnlichkeiten. So dominiert sowohl auf Seiten der Lehrpersonen als auch auf Seiten der Schüler*innen die Position der mehr (Schüler*innen) oder minder (Lehrpersonen) motivierten Nutzer*innen (Abschn. 5b). Als erstrebenswerte Norm wird im Schreiben der Grundschullehrer*innen die Position kritischreflexiver, aber vor allem produktiver Subjekte konstituiert, wobei letztere weniger im Sinne der Figur der „Maker“ (Macgilchrist 2017a) agieren, als vielmehr unterrichtliche Produkte - wie beispielsweise Präsentationen (vgl. Verena 2018b) oder Lexikonartikel (Bongardt 2003; Daugs 2004) - erstellen. Darüber hinaus werden eine Vielzahl anderer - von den Analysen Macgilchrists abweichender bzw. diese ergänzender - Subjektpositionen entworfen (Abschn. 5b). Die Positionierung der schreibenden Grundschullehrer*innen weist selbige nicht zuletzt als ebenso mutige wie Orientierung vermittelnde Avantgarde einer ,zukunftsweisenden“ (Gach 2018) Entwicklung aus und an anderer Stelle als Spielleiter*innen einer gamifizierten und technologisierten Unterrichtspraxis (Höhne 2015; Buck 2017), in der die generalisierten Schüler*innensubjekte stets mit Freude lernen, sich selbst zu steuern.

\section{Und zum Schluss: (Forschungs-)Fragen}

Die in diesem Artikel präsentierten Analyseergebnisse werfen eine Reihe von Fragen auf, denen es weiter nachzugehen lohnt. So stellt sich unter anderem die Frage, wie die rekonstruierten Begründungsfiguren und Positionierungen im Schreiben über das Lehren und Lernen mit digitalen Medien sich zu unterrichtlichen Praktiken und damit hervorgebrachten Positionierungen verhalten. Werden in unterrichtlichen Praktiken ähnliche Normen konstituiert? Wo lassen sich Verschiebungen beobachten? Inwiefern variieren die insbesondere mit Bezug auf Schüler*innen vorgenommenen generalisierten Subjektpositionen? Was gerät aus dem Blick, wenn Schule wie von den schreibenden Grundschullehrer*innen entworfen - als unter Nutzung digitaler Medien optimierbar und entwicklungsbedürftig gerahmt wird? Zukünftige Forschung könnte zeigen, welchen feldübergreifenden Iterationen die im Schreiben 
über das Lehren und Lernen mit digitalen Medien hervorgebrachten Normen und Subjektpositionen unterliegen.

Funding Open Access funding provided by Projekt DEAL.

Open Access Dieser Artikel wird unter der Creative Commons Namensnennung 4.0 International Lizenz veröffentlicht, welche die Nutzung, Vervielfältigung, Bearbeitung, Verbreitung und Wiedergabe in jeglichem Medium und Format erlaubt, sofern Sie den/die ursprünglichen Autor(en) und die Quelle ordnungsgemäß nennen, einen Link zur Creative Commons Lizenz beifügen und angeben, ob Änderungen vorgenommen wurden.

Die in diesem Artikel enthaltenen Bilder und sonstiges Drittmaterial unterliegen ebenfalls der genannten Creative Commons Lizenz, sofern sich aus der Abbildungslegende nichts anderes ergibt. Sofern das betreffende Material nicht unter der genannten Creative Commons Lizenz steht und die betreffende Handlung nicht nach gesetzlichen Vorschriften erlaubt ist, ist für die oben aufgeführten Weiterverwendungen des Materials die Einwilligung des jeweiligen Rechteinhabers einzuholen.

Weitere Details zur Lizenz entnehmen Sie bitte der Lizenzinformation auf http://creativecommons.org/ licenses/by/4.0/deed.de.

\section{Literatur}

Bastian, J. (2017). Lernen mit Medien - Lernen über Medien? Eine Bestandsaufnahme zu aktuellen Schwerpunktsetzungen. DDS - Die Deutsche Schule, 102(2), 146-162.

Bastian, J., \& Aufenanger, S. (Hrsg.). (2017). Tablets in Schule und Unterricht. Forschungsmethoden und Perspektiven zum Einsatz digitaler Medien. Wiesbaden: Springer VS.

Bongardt, E. (2003). Beiträge von Kindern für Kinder im Internet. Praxis Grundschule, 35(1), 18-21.

Buck, M.F. (2017). Gamification von Unterricht als Destruktion von Schule und Lehrberuf. Vierteljahresschrift für wissenschaftliche Pädagogik, 93(2), 268-282.

Bundesministerium für Bildung und Forschung (BMBF) (2019). Digitale Zukunft. Lernen. Forschen. Wissen. Die Digitalstrategie des BMBF. Berlin. https://www.bmbf.de/upload_filestore/pub/BMBF_ Digitalstrategie.pdf. Zugegriffen: 3. Juni 2019.

Daugs, H. (2004). Interessenbezogenes Schülerlexikon. Praxis Grundschule, 36(3), 5-20.

Gach, H. J. (2018). Digitale Medien gestern und heute. Praxis Grundschule, 50(3), 6-8.

Grömping, M., \& Schwarze, D. (2003). Internetpräsenz: Motivation für die Schüler - Information für die Eltern. Praxis Grundschule, 35(1), 10-11.

Grundschule (2019). Anzeigen-Tarif und Media-Informationen. https://www.westermann.de/ produktfamilie/GRU/Grundschule-Ihre-verlaessliche-Partnerin\#Mediadaten. Zugegriffen: 3. Juni 2019.

Halbach, M. (2003). Medienkonzept im Schulprogramm. Begründungen zum Einsatz von Computern. Praxis Grundschule, 35(1), 24-29.

Höhne, T. (2015). Technologisierung von Bildungsmedien. DDS - Die Deutsche Schule, 107(1), 8-19.

Johnson, N.F. (2019). Dysfunctional devices in the classroom meet the habitus of the new. E-Learning and Digital Media, 16(3), 208-220. https://doi.org/10.1177/2042753019831385.

Klöckener, J. (2013). Bewertung von Lernsoftware für den Mathematikunterricht. Praxis Grundschule, $45(5), 50-53$.

Klomfaß, S., \& Moldenhauer, A. (2018). Kontrollierte Freisetzung? Eine Diskursanalyse von Darstellungen individualisierten Unterrichts aus der Zeitschrift PÄDAGOGIK. In K. Rabenstein, K. Kunze, M. Martens, T.-S. Idel, M. Proske \& S. Strauß (Hrsg.), Individualisierung von Unterricht. Transformationen - Wirkungen - Praxis (S. 214-230). Bad Heilbrunn: Julius Klinkhardt.

Krause, H.-J. (2007). Der Computer als Werkzeug für die Rechtschreibung. Praxis Grundschule, 39(4), $22-23$.

Kultusministerkonferenz der Länder (KMK) (2016). Bildung in der digitalen Welt. Strategie der Kultusministerkonferenz. In der Fassung vom 07.12.2017. https://www.kmk.org/fileadmin/Dateien/pdf/ PresseUndAktuelles/2017/Strategie_neu_2017_datum_1.pdf. Zugegriffen: 7. Febr. 2019.

Luhmann, N., \& Schorr, K.-E. (1982). Das Technologiedefizit der Erziehung und die Pädagogik. In N. Luhmann \& K.-E. Schorr (Hrsg.), Technologie und Selbstreferenz. Fragen an die Pädagogik (S. 11-40). Frankfurt am Main: Suhrkamp. 
Macgilchrist, F. (2017a). Die medialen Subjekte des 21. Jahrhunderts: Digitale Kompetenzen und/oder Critical Digital Citizenship. In H. Allert, M. Asmussen \& C. Richter (Hrsg.), Digitalität und das Selbst. Interdisziplinäre Perspektiven auf Subjektivierungs- und Bildungsprozesse (S. 145-168). Bielefeld: transcript.

Macgilchrist, F. (2017b). Backstaging the teacher: On learner-driven school-driven and data-driven change in educational technology discourse. Kultura - Społeczeństwo - Edukacja, 2(12), 83-103. https://doi. org/10.14746/kse.2017.12.4.

Matthes, E., Schütze, S., \& Wiater, W. (Hrsg.). (2013). Digitale Bildungsmedien im Unterricht. Bad Heilbrunn: Klinkhardt.

Maurer, S. (2016). Empfehlungen einer Kollegin: Apps für den Unterricht. Grundschule, 48(5), 34.

Mayrberger, K. (2007). Verändertes Lernen mit neuen Medien? Strukturanalyse gemeinschaftlicher Interaktionen in einer computergestützen Lernumgebung in der Grundschule. Hamburg: Verlag Dr. Kovac.

Nuxoll, F. (2018). Digitale Bildung als Aufgabe nehmen. Grundschule, 50(7), 38-39.

Peck, C., Kappler Hewitt, K., Mullen, C. A., Lashley, C., Eldrigde, J., \& Douglas, T. O. (2015). Digital youth in brick and mortar schools: examining the complex interplay of students, technology, education, and change. Teacher College Record, 117(5), 1-40.

Pereira, S., \& Pereira, L. (2015). Digital media in primary schools: literacy or technology? Analyzing government and media discourses. Educational Policy, 29(2), 316-341. https://doi.org/10.1177/ 0895904813492378.

Peschel, F., \& Irion, T. (Hrsg.). (2016). Neue Medien in der Grundschule 2.0. Grundlagen - Konzepte Perspektiven. Frankfurt a.M.: Grundschulverband.

Philip, T.M., \& Garcia, A.D. (2013). The importance of still teaching the igeneration: new technologies and the centrality of pedagogy. Harvard Educational Review, 83, 300-319.

Player-Koro, C., Bergviken Rensfeldt, A., \& Selwyn, N. (2018). Selling tech to teachers: education trade shows as policy events. Journal of Education Policy, 33(5), 682-703. https://doi.org/10.1080/ 02680939.2017.1380232.

Praxis Grundschule (2019). Anzeigen-Tarif und Media-Informationen. https://www.westermann.de/ produktfamilie/PGRU/Praxis-Grundschule\#Mediadaten. Zugegriffen: 3. Juni 2019.

Proske, M., \& Niessen, A. (2017). Medialität und Performativität im Unterricht: Zwischen Hervorbringen und Übertragen, Inszenieren und Wahrnehmbarmachen schulischen Wissens, Könnens und Sollens. ZISU - Zeitschrift für interpretative Schul- und Unterrichtsforschung, 6, 3-13.

Rabenstein, K., \& Reh, S. (2009). Die pädagogische Normalisierung der ,selbstständigen Schülerin“ und die Pathologisierung des ,Unaufmerksamen“. Eine diskursanalytische Skizze. In J. Bilstein \& J. Ecarius (Hrsg.), Standardisierung - Kanonisierung. Erziehungswissenschaftliche Reflexionen (S. 159-180). Wiesbaden: Springer VS.

Sawischlewski, A. (2003). Kennst Du Dich mit dem Computer aus? Praxis Grundschule, 35(3), $58-64$.

Schaumburg, H., \& Prasse, D. (2019). Medien und Schule. Theorie - Forschung - Praxis. Bad Heilbrunn: Klinkhardt.

Schmidt, M., \& Herfter, C. (2019). Die Kultur der Aufgabenkultur. Eine Analyse der Praktiken der Adressierung von Lehrkräften in praxisinstruktiven Zeitschriften. Zeitschrift für Grundschulforschung. https://doi.org/10.1007/s42278-019-00039-6.

Seidel, T. (2011). Digitale Klassenräume. Das interaktive Whiteboard im Grundschulunterricht. Grundschule, 43(12), 30-33.

Selwyn, N. (2013). Rethinking education in the digital age. In I. K. Orton-Johnson \& N. Prior (Hrsg.), Digital sociology. Critical perspectives (S. 197-212). New York: Palgrave Macmillan.

Selwyn, N., Nemorin, S., Bulfin, S., \& Johnson, N. (2016). Toward a digital sociology of school. In J. Daniels, K. Gregory \& M. M. T. Cottom (Hrsg.), Digital sociologies (S. 147-162). Bristol: Policy Press.

Stolz, N. (2012). Der Computer: Freund und Helfer. Elektronische Medien bilden die Lebenswirklichkeit unserer Kinder ab. Grundschule, 44(2), 27-29.

Tenorth, H.-E. (2017). Reformpädagogik - oder: die Modernisierung der Erziehung. In T.-S. Idel \& H. Ullrich (Hrsg.), Handbuch Reformpädagogik (S. 22-45). Weinheim: Beltz Juventa.

Thielbeer, R. (2018). Symmetrie mit Tablets und Co. Praxis Grundschule, 50(7), 20-23.

Thon, C. (2014). Individualisierung im Unterricht. Praxishilfen für Lehrer_innen als Anrufungsanleitungen. In B. Kleiner \& N. Rose (Hrsg.), (Re-)Produktion von Ungleichheit im Schulalltag. Judith Butlers Konzept der Subjektivation in der erziehungswissenschaftlichen Forschung (S. 155-171). Opladen: Barbara Budrich.

Verena, (2018a). Grundschulschnüffler. Über Leben im Schulalltag. Mein neues Projekt Tabletklasse - Es geht los. Grundschule, 50(7), 46-47. 
Verena (2018b). Grundschulschnüffler. Über Leben im Schulalltag. Mein neues Projekt Tabletklasse Präsentieren lernen. Grundschule, 50(8), 46-47.

Wrana, D. (2006). Das Subjekt schreiben. Reflexive Praktiken und Subjektivierung in der Weiterbildung. Baltmannsweiler: Schneider-Verlag Hohengehren.

Wrana, D. (2015a). Zur Methodik einer Analyse diskursiver Praktiken. In F. Schäfer, A. Daniel \& F. Hillebrandt (Hrsg.), Methoden einer Soziologie der Praxis (S. 121-144). Bielefeld: transcript.

Wrana, D. (2015b). Zur Analyse von Positionierungen in diskursiven Praktiken. Methodologische Reflexionen anhand von zwei Studien. In I. S. Fegter, F. Kessl, A. Langer, M. Ott, D. Rothe \& D. Wrana (Hrsg.), Erziehungswissenschaftliche Diskursforschung. Empirische Analysen zu Bildungs- und Erziehungsverhältnissen (S. 123-142). Wiesbaden: Springer VS. 